\title{
Aplicación del Aprendizaje Basado en Problemas (ABP) en una asignatura del Máster Universitario de Ingeniería Industrial: propuesta metodológica y de evaluación
}

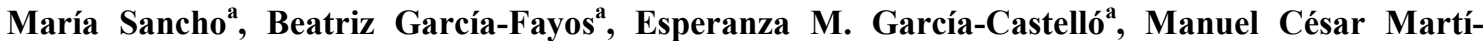
Calatayud $^{a}$, Antonio D. Rodríguez-López ${ }^{\mathrm{a}}$, Amparo Bes-Pia ${ }^{\mathrm{a}}$, José Antonio Mendoza-Roca ${ }^{\mathrm{a}}$ y Asunción Santafé-Moros ${ }^{\mathrm{a}}$

${ }^{a}$ Departamento de Ingeniería Química y Nuclear, Universitat Politècnica de València, emails: msanchof@iqn.upv.es; beagarfa@iqn.upv.es; egarcia1@iqn.upv.es; mcmarti@iqn.upv.es; anrodlo@iqn.upv.es; mbespia@iqn.upv.es; jamendoz@iqn.upv.es; assanmo@iqn.upv.es

\begin{abstract}
In the subject "Industrial Chemical Technology" of the Master's Degree in Industrial Engineering of the Polytechnic University of Valencia (UPV) content related to the Basic Operations of Chemical Engineering is taught, not previously studied by the most students. This fact, together with the complications associated with the average of 300 students that the subject has, conditions the type of methodologies that can be applied and, therefore, the level of depth at which the contents can be developed. Therefore, the main methodology applied so far has been the participatory master lesson, with problem solving in the classroom. Although the results in terms of percentage of students who pass the subject are very satisfactory, the learning approach is very superficial and we believe that it should be more adapted to the master's level of the subject. To achieve a deeper learning, it has been decided to partially modify the methodology of the subject, integrating the Problem Based Learning (BPL). This work describes how the BPL methodology will be implemented and evaluated in the subject. All this is part of an Innovation and Educational Improvement Project granted by the VECA of the UPV.
\end{abstract}

Keywords: deep learning, methodology, Problem Based Learning, chemical technology, industrial engineering

\section{Resumen}

En la asignatura "Tecnología Química Industrial" del Máster Universitario en Ingeniería Industrial de la Universitat Politécnica de València (UPV) se imparten contenidos relativos a las Operaciones Básicas de la Ingeniería Química, no estudiados previamente por la mayoría de los alumnos. Este hecho, unido a las complicaciones asociadas a la media de 300 alumnos que tiene la asignatura, condiciona el tipo de metodologías que se pueden aplicar y, por lo tanto, el nivel de profundidad en el que se pueden desarrollar los contenidos. Por ello, la metodología principal aplicada hasta el momento ha sido la lección magistral participativa, con resolución de problemas en el aula. Aunque los resultados en cuanto a porcentaje de alumnos que superan la asignatura son muy satisfactorios, el enfoque de aprendizaje es muy superficial y creemos que debería adecuarse más al nivel de máster de la asignatura. Para conseguir un aprendizaje más profundo, se ha decido modificar parcialmente la metodología de la asignatura, integrando el Aprendizaje Basado en Problemas (ABP). En este trabajo se describe cómo se va a implementar y evaluar la metodología ABP en la asignatura. Todo ello forma parte de un Proyecto de Innovación y Mejora Educativa concedido por el VECA de la UPV. 
Palabras clave aprendizaje profundo, metodología, Aprendizaje Basado en Problemas, tecnología química, ingeniería industrial

\section{Introducción}

Este trabajo se engloba dentro un Proyecto de Innovación y Mejora Educativa (PIME) solicitado y concedido en el año 2019, para integrar el Aprendizaje Basado en Problemas en una asignatura del Máster Universitario en Ingeniería Industrial de la Universitat Politècnica de València (UPV). El Proyecto tiene una duración de dos cursos académicos: el primero (2019-20), en el que se definirá el cambio metodológico a realizar, la creación de contenidos y las modificaciones necesarias que implique esta innovación en el sistema de evaluación; y el segundo (2020-21), en el que se implementará la innovación en la asignatura. El presente artículo recoge los logros en el desarrollo del Proyecto alcanzados tras el primer curso de ejecución (2019-20), que han consistido en la definición de la metodología a implementar, con los correspondientes cambios en el sistema de evaluación, así como en la elaboración de los materiales docentes necesarios.

\subsection{Contexto de la asignatura}

La asignatura objeto del PIME es “Tecnología Química Industrial”, una asignatura troncal del $1^{\text {er }}$ curso del Máster Universitario en Ingeniería Industrial de la Escuela Técnica Superior de Ingeniería Industrial de la UPV que se imparte desde el curso 2014-2015. La asignatura consta de 4,5 créditos ECTS, 3,6 de los cuales son de teoría y práctica de aula, y los restantes 0,9 de prácticas de laboratorio y prácticas informáticas. La asignatura cuenta con unos 300 alumnos aproximadamente que, desde el curso 2019-20, están estructurados en 8 grupos de teoría y 24 grupos de prácticas. La parte teórica de la asignatura es impartida por un total de 8 profesores, algunos de los cuales imparten un grupo completo, si bien otros, por cuestiones de compatibilidad con otras asignaturas, sólo imparten uno de los dos parciales en uno o varios grupos.

Respecto a la estructura de los contenidos de la asignatura, éstos se encuentran divididos en las unidades didácticas que se muestran en la Tabla 1.

Tabla 1. Unidades didácticas de la asignatura

\begin{tabular}{cll}
\hline $\begin{array}{c}\text { Unidad } \\
\text { didáctica }\end{array}$ & Nombre & Prácticas \\
\hline 1 & Introducción a los Procesos Químicos Industriales & \\
2 & Balances en Ingeniería Química & Balance de Materia en Estado No Estacionario \\
3 & Cinética y Reactores & \\
4 & La Planta Química & Destilación Diferencial de una mezcla binaria \\
\hline
\end{tabular}

De las 5 unidades didácticas, las 3 primeras se evalúan en el primer parcial de la asignatura (tras 6 semanas de clase) y las 2 siguientes en el segundo parcial (tras otras 6 semanas de clase). En cada bloque de 6 semanas lectivas los alumnos realizan, en grupos de 3 a 5 miembros, además una práctica de laboratorio y una informática sobre un tema de la misma unidad didáctica, tal y como se indica en la 
Tabla 1. Como resultado de estas prácticas, los alumnos entregan un informe escrito por grupo, en el que presentan y discuten los resultados experimentales.

Respecto a la metodología aplicada en la asignatura, está basada en la lección magistral participativa con resolución de problemas en aula, además de las prácticas de laboratorio e informáticas realizadas. Mediante dicha metodología se desarrollan las competencias específicas correspondientes, además de las tres competencias transversales que la asignatura tiene asignadas como punto de control: la CT1 "Comprensión e integración”, la CT3 "Análisis y resolución de problemas" y la CT6 "Trabajo en equipo y liderazgo". La CT1 y la CT6 se desarrollan y evalúan a través de las prácticas de laboratorio e informáticas, mientras que la CT3 se desarrolla a partir de la resolución de problemas en aula y es evaluada a través de los exámenes escritos.

Finalmente, en la evaluación de la asignatura se incluye la nota de cada examen parcial (45\%) y la nota de los informes de prácticas ( $5 \%$ cada uno). El examen parcial consta de un test de unas 12 preguntas con opción múltiple, que supone un $35 \%$ de la nota del examen, y uno o dos problemas, que representan el restante $65 \%$.

\subsubsection{Justificación de la innovación planteada}

La metodología actual aplicada en la asignatura ha estado condicionada por una serie de dificultades encontradas a lo largo de los cursos académicos. Son las siguientes:

- el hecho de ser una asignatura de nueva creación que no existía en el plan de estudios anterior;

- el aumento constante de alumnos desde el inicio de los nuevos Másteres (desde 164 el primer año hasta los 289 del curso actual, pasando por 385 en el curso 2018-19);

- el aumento de grupos de teoría (de 3 el primer año hasta los 8 del curso actual), con la necesidad de incorporación de nuevos profesores todos los cursos.

Por otra parte, la asignatura contempla la formación en las Operaciones Básicas de la Ingeniería Química (Operaciones de Separación y Reactores Químicos), disciplinas no estudiadas por los alumnos durante el Grado en Ingeniería en Tecnologías Industriales, titulación de la que proceden mayoritariamente los alumnos que acceden al Máster, lo que limita también el tipo de actividades que es posible realizar. Por todo ello, se optó por una metodología tradicional que hasta la fecha ha funcionado correctamente dado que el porcentaje de aprobados de la asignatura es muy elevado: superior al $90 \%$ en todos los cursos.

Sin embargo, existen algunos motivos para plantearse modificar la metodología de la asignatura. Por un lado, a pesar de los buenos resultados académicos obtenidos por los estudiantes, los profesores de teoría compartimos que el aprendizaje de los alumnos resulta poco profundo debido a que los problemas que se resuelven están planteados como unidades independientes para cada unidad, no consiguiendo que se adquiera una visión global de los procesos químicos industriales, tal y como sería deseable. Por otro lado, la evaluación de las competencias transversales CT1 y CT3 está demasiado centrada en los problemas de examen, lo cual no es la manera más adecuada de trabajar ni evaluar su grado de adquisición. Además, los procesos de acreditación del Máster (tanto ABET como EURACE), han evidenciado que la asignatura es una de las de $1^{\mathrm{er}}$ curso que menos actividades evaluables realizaba al margen de exámenes y prácticas.

Por todo ello, el curso pasado se solicitó un Proyecto de Innovación y Mejora Educativa con el fin de replantear parte de la metodología de la asignatura para mejorar el aprendizaje de los estudiantes y darle un enfoque más profundo y acorde al nivel de Máster de la asignatura. 


\subsection{Estrategias para un aprendizaje profundo}

Los enfoques de aprendizaje se refieren a la manera como los estudiantes se enfrentan a una tarea académica. Estos enfoques derivan tanto de las percepciones que el estudiante tiene de la tarea como de sus características personales (Biggs, 1993), pudiéndose distinguir básicamente dos enfoques: superficial y profundo. El aprendizaje superficial se caracteriza por la memorización pasiva de conocimiento, enfocada a la consecución de la tarea. En este enfoque el aprendizaje se aprecia como una imposición que resulta en una desconexión entre la nueva información y el conocimiento ya adquirido. Por su parte, el enfoque profundo de aprendizaje se caracteriza por la interacción de las nuevas ideas y conceptos adquiridos con el conocimiento previo, así como por el análisis crítico de los resultados. En este enfoque, el estudiante es consciente de su propio proceso de aprendizaje y su motivación radica precisamente en dicho proceso (Wai Lu, 2015).

Está comprobado que existe una conexión entre el método de enseñanza y la profundidad y la complejidad del aprendizaje. De hecho, los alumnos aprenden de una manera determinada según la forma en que se les enseña y evalúa, pudiendo modificar su enfoque de aprendizaje mediante planteamientos metodológicos basados en el aprendizaje (Gargallo, 2008). Así, en comparación con los métodos tradicionales de enseñanza, las metodologías activas suponen una mayor implicación de los estudiantes en su propio proceso de aprendizaje, lo que resulta en un nivel de comprensión superior, incrementando su enfoque profundo de aprendizaje y reduciendo el superficial (Gargallo López, 2015 \& Constantino, 2019).

Una de las metodologías centradas en el aprendizaje activo es el Aprendizaje Basado en Problemas (De Graaff, 2003). Se trata de un método centrado en el estudiante, basado en el uso de problemas como el punto de partida para la adquisición de nuevos conocimientos. Dado que la asignatura en la que se pretende replantear la metodología está basada principalmente en la resolución de problemas, se ha decidido que ésta será la nueva metodología a implementar para lograr un aprendizaje más profundo de nuestros estudiantes. Para ello, habrá que tener en cuenta las principales características que debe cumplir la metodología ABP (Pérez Granado, 2018):

- $\quad$ El proceso se centra en el estudiante, quien asume el control de su propio aprendizaje.

- $\quad$ En papel del docente es como tutor y facilitador del aprendizaje.

- Los problemas son la base para alcanzar los resultados de aprendizaje de la materia.

- $\quad$ Se debe realizar en equipos de trabajo de máximo 10 miembros.

- $\quad$ El trabajo debe ser colaborativo.

Esta metodología ha experimentado algunos cambios al ser adoptada por distintas instituciones, siendo la de la universidad de Maastricht, por ejemplo, una de las más extendidas, especialmente en Europa. Una de las variables principales del ABP tiene que ver con el grado de estructuración del problema, pudiendo encontrar desde problemas rígidamente estructurados y con alto grado de detalles, hasta problemas abiertos que no presentan datos y en los que queda en manos del estudiante la investigación del problema y, en cierta medida, su definición (Vizcano Guarch, 2008).

En cualquiera de sus variantes, diversos estudios han demostrado que el Aprendizaje Basado en Problemas tiene un efecto de mejora en el enfoque de aprendizaje de los estudiantes. Así, se ha comprobado que esta estrategia tiene un efecto positivo en el aprendizaje procedimental de los estudiantes, así como en la disminución de la carga mental durante el proceso de aprendizaje (Bayat, 2012). Además, se ha demostrado que esta metodología juega un papel importante en la permanencia del conocimiento (Benli, 2012). 
Sin embargo, hay que tener en cuenta que cuando los estudiantes se enfrentan a esta estrategia por primera vez muestran cierta resistencia y frustración en las primeras fases, ante el desconocimiento del modo en que tienen que desarrollar las tareas y gestionar su propio aprendizaje. Ante esta situación, la motivación del estudiante hacia la actividad es fundamental para desarrollar con éxito los objetivos de aprendizaje. Entre las estrategias de motivación que permiten el éxito del ABP se encuentran, por un lado, la contextualización de los problemas, procurando que sean lo más realistas posibles, acercando al estudiante a su futuro profesional (Farida Harun, 2012); y, por otro lado, es muy importante la acción tutorial de guía y acompañamiento por parte del profesor (Perrenet, 2000).

\subsubsection{Aplicación del ABP en ingeniería}

Del análisis de la literatura existente en relación a la resolución de problemas en ingeniería, se ha encontrado que algunos autores afirman que no es posible alcanzar un aprendizaje profundo en dicha competencia a través de las metodologías convencionales. En varios trabajos consultados se sugiere la metodología de Aprendizaje Basado en Problemas (ABP) como una de las mejores estrategias para la formación en ingeniería (Wai Hung, 2003). La justificación de ello se basa en el carácter multidisciplinar de esta metodología, en el desarrollo que permite de competencias de alto nivel, así como del trabajo en equipo y de habilidades comunicativas. Además, en la literatura se recomienda que los problemas planteados estén basados en situaciones reales, ya que de este modo la formación a través de esta metodología permite reducir la brecha entre lo que se enseña y lo que la industria espera de los egresados (Lau, 2004). Dado que se trata de una metodología que se basa en establecer relaciones entre la nueva información y el conocimiento previo de los estudiantes, el aprendizaje adquirido es más útil y más fácilmente transferible a contextos profesionales (Perrenet, 2000).

Además, el Aprendizaje Basado en Problemas permite no solo adquirir competencias cognitivas, sino también una serie de habilidaddes y competencias transversales (Barrow, 1986 \& Draghicescu, 2014). Así, proporciona a los estudiantes la oportunidad de trabajar en grupo y adquirir experiencia en el trabajo en equipo, tan necesario para el desarrollo profesional de los ingenieros. Asimismo, esta metodología permite desarrollar el autoaprendizaje del estudiante, ya que se requiere la búsqueda y selección de información de manera eficiente para su aplicación en la resolución del problema planteado (Drobnic Vidic, 2008).

\section{Objetivos}

El objetivo principal de este trabajo es definir el nuevo contexto metodológico y de evaluación de la asignatura para la integración del Aprendizaje Basado en Problemas. Para ello, se establecen los siguientes objetivos específicos:

- Definir la nueva metodología de la asignatura basada en el ABP, incluyendo la organización de los alumnos ( $\mathrm{n}^{\mathrm{o}}$ de grupos, $\mathrm{n}^{\mathrm{o}}$ de miembros por grupo, etc.) y el tipo de actividades a realizar.

- Establecer el plan de evaluación y seguimiento de la nueva metodología, considerando la planificación de la acción tutorial.

- Diseñar los problemas en los que se basará el ABP. 


\section{Desarrollo de la innovación}

Para el desarrollo de los objetivos mencionados se han llevado a cabo una serie de reuniones entre los profesores de teoría de la asignatura, y se han planteado y ejecutado diferentes tareas, tal cual se muestra en la Figura 1.

En primer lugar, se realizó una reunión inicial con todo el profesorado implicado para coordinar y plantear las tareas a realizar durante el curso 2019-20. Las primeras tareas planteadas fueron las siguientes:

- Definir el modo de aplicación de la metodología ABP en la asignatura, para lo cual había que plantearse y decidir cómo se organizarán los alumnos para realizar las diferentes tareas, así como la planificación temporal de las distintas actividades que realizarán.

- Analizar las distintas alternativas de evaluación, fundamentalmente qué herramientas se utilizarán para ello y cómo se verán modificados los criterios de evaluación actuales. Esta tarea incluía además la definición de la acción tutorial para guiar y reportar feed-back sobre el trabajo a los estudiantes.

Estas dos tareas derivaron posteriormente en una reunión de puesta en común en la que se acordaron y definieron tanto la metodología como la evaluación de la asignatura con la incorporación del ABP. Todas las modificaciones establecidas respecto al curso anterior, serán incorporadas en la Guía Docente de la asignatura en el plazo correspondiente.

En paralelo a las tareas anteriores, se planteó la definición del contexto en el que se desarrollará el ABP. Para ello, se decidió que cada profesor realizaría varias propuestas de procesos industriales en los que se pudieran plantear problemas relacionados con los conceptos de tecnología química estudiados en las unidades didácticas de la asignatura. A partir de las propuestas, se realizó una puesta en común que concluyó con la selección de algunos procesos. Posteriormente, para cada proceso se han planteado distintos enfoques de problemas, de manera que de cada proceso se puedan extraer varios problemas distintos relacionados con una o varias unidades didácticas. Finalmente, se redactarán las actividades con los problemas, tal cual se les vayan a proporcionar a los alumnos, antes de que finalice el curso actual.

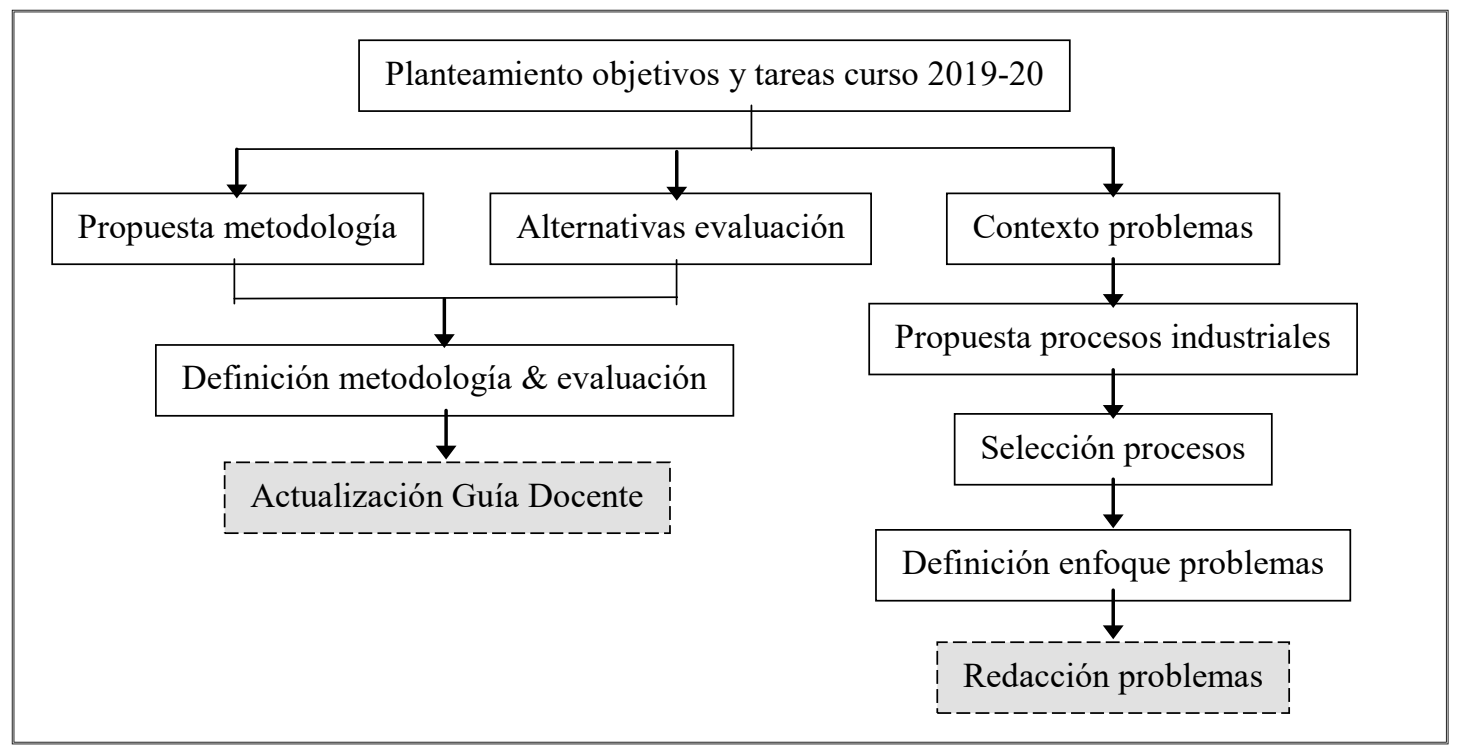

Fig. 1 Secuencia de desarrollo de la innovación durante el curso 2019-20 


\section{Resultados}

\subsection{Definición de la metodología}

En primer lugar, cabe señalar que los profesores de la asignatura acordamos aplicar una adaptación de la metodología de Aprendizaje Basado en Problemas con problemas parcialmente estructurados, en consonancia con el contexto de la asignatura, ya que los conceptos teóricos que se imparten han sido adaptados para la asignatura por los profesores de la misma a partir de la bibliografía específica de Ingeniería Química, no habiéndose encontrado literatura adaptada al nivel requerido para los estudiantes de esta asignaura. Así, los conocimientos teóricos básicos para la realización de los problemas serán impartidos por el profesorado en las clases de teoría, si bien el alcance de los resultados requeridos en los problemas del ABP y su relación entre conceptos o con otras disciplinas precisará de la investigación y aprendizaje autónomo por parte de los estudiantes, de manera que trabajen de modo inductivo los resultados de aprendizaje que les lleven a adquirir un enfoque más profundo.

En relación al modo de aplicación de la metodología $\mathrm{ABP}$ en la asignatura, se acordaron las siguientes decisiones:

- La actividad será obligatoria para todos los alumnos, y se realizará principalmente de manera no presencial. Esto facilitará que los alumnos que no pueden asistir a clase por motivos laborales puedan llevar a cabo el ABP y adquirir también un mejor aprendizaje.

- Los alumnos de cada grupo de teoría se dividirán en equipos de máximo 4 alumnos. Dado que la asignatura está estructurada en 8 grupos de teoría, y la media de matriculados es de unos 300 estudiantes, se estiman un total de 8-10 equipos de trabajo por clase (suponiendo máximo 45 alumnos por grupo).

- La metodología ABP a realizar se explicará en la primera sesión de clase de la asignatura. Los alumnos dispondrán de toda la información al respecto en el PoliformaT.

- La formación de los grupos se llevará a cabo de manera libre a través de la herramienta de PoliformaT, y se les dará de plazo hasta la $2^{\mathrm{a}}$ semana desde el inicio de las clases.

- Los problemas a resolver por cada equipo estarán relacionados con un mismo proceso industrial para que puedan adquirir una visión global del mismo.

- El enfoque de los problemas debe incluir consideraciones no sólo de tipo técnico sino también económico y de sostenibilidad, y debe procurarse que tenga cierto carácter multidisciplinar. De esta forma, además de que los alumnos adquieran una visión global de la complejidad de un proceso industrial químicos, se pretende que comprendan el papel importante de la industria química en la sociedad (Lennon, 2002).

- Los problemas planteados deberán requerir la búsqueda por parte de los alumnos de algún tipo de información: legislación, normativa, propiedades físico-químicas, etc. De este modo, se desarrollarán las competencias relacionadas con la búsqueda y selección crítica de información.

- Los alumnos resolverán un problema en cada parcial. En el primero, estará relacionado con la unidad didáctica 2, evitándose la número 3, para que puedan desarrollarlo con tiempo suficiente antes del examen parcial. El problema a resolver durante la segunda parte de la asignatura podrá estar relacionado con cualquiera de las dos unidades didácticas, ya que en este caso hay más tiempo para su resolución al ser el examen del segundo parcial a mediados o finales del mes de enero. En la Tabla 2 se puede observar la distribución temporal de las unidades didácticas a lo largo del cuatrimestre.

\subsection{Definición de evaluación y seguimiento}

Como producto de la actividad, el grupo de profesores de la asignatura ha decidido que consista en un informe escrito con los 3 entregables siguientes:

- Entregable 1. Descripción del contexto del proceso industrial asignado, incluyendo un diagrama de bloques del mismo. Fecha aproximada de entrega: semana 3 del cuatrimestre. 
- Entregable 2. Resolución del problema de la Unidad Didáctica 2. Fecha aproximada de entrega: mitad del cuatrimestre (antes del primer examen parcial).

- Entregable 3. Resolución del problema de las Unidades Didácticas 4 y/o 5. Fecha aproximada de entrega: final cuatrimestre (antes del segundo examen parcial).

Para el seguimiento del trabajo y poder proporcionarles feed-back se ha decidido que cada equipo de trabajo deberá enviar al profesor correspondiente una ficha en formato tabla que incluirá el enfoque de resolución previsto y la información seleccionada, antes de que pasen a resolver los problemas.

En la Tabla 2 se muestra gráficamente la programación temporal de la metodología y evaluación planteadas, incluyendo las entregas de las fichas de feed-back.

Tabla 2. Programación temporal de la asignatura con el ABP

\begin{tabular}{|c|c|c|c|c|c|c|c|}
\hline Semana & 1 & 2 & 3 & 4 & 5 & 6 & $\begin{array}{l}\text { Examen } \\
\text { Parcial } 1\end{array}$ \\
\hline Fechas aprox. & $22 \mathrm{sept}$ & & & & & & $4-17$ nov \\
\hline $\begin{array}{l}\text { Programación } \\
\text { asignatura }\end{array}$ & $\begin{array}{l}\text { Presentación } \\
\text { + UD1 }\end{array}$ & UD2 & UD2 & UD3 & UD3 & UD3 & \\
\hline Hito ABP & $\begin{array}{l}\text { Explicación } \\
\text { ABP }\end{array}$ & $\begin{array}{l}\text { Fin formación } \\
\text { equipos }\end{array}$ & Entregable 1 & $\begin{array}{l}\text { Ficha } 1 \\
\text { feed-back }\end{array}$ & & Entregable 2 & \\
\hline Semana & 7 & 8 & 9 & 10 & 11 & 12 & $\begin{array}{l}\text { Examen } \\
\text { Parcial } 2\end{array}$ \\
\hline Fechas aprox. & 18 nov & & & & & 11 enero & $\begin{array}{l}18-29 \\
\text { ene }\end{array}$ \\
\hline $\begin{array}{l}\text { Programación } \\
\text { asignatura }\end{array}$ & UD4 & UD4 & UD5 & UD5 & UD5 & UD5 & \\
\hline Hito ABP & & & $\begin{array}{l}\text { Ficha } 2 \\
\text { feed-back }\end{array}$ & & & Entregable 3 & \\
\hline
\end{tabular}

La actividad supondrá un $10 \%$ de peso en la calificación final. Dado que es una actividad obligatoria, el estudiante que no la realice tendrá una calificación de 0 en esa actividad y no se podrá recuperar.

\subsection{Propuesta de procesos industriales para la aplicación del ABP}

De cara a la propuesta de procesos industriales para la aplicación de la metodología ABP se plantearon los siguientes condicionantes:

- Debían ser procesos relacionados con la industria química, susceptibles de integrar los conocimientos básicos estudiados en la asignatura pero que permitan la aplicación y relación de otros conocimientos que deban ser trabajados de forma autónoma por los estudiantes.

- Debían ser procesos que resultaran motivadores para los estudiantes, teniendo en cuenta que su formación previa es mayoritariamente del Grado en Ingeniería en Tecnologías Industriales, evitando procesos con un enfoque puramente químico.

En total se propusieron 12 procesos industriales, de los cuales se acordó seleccionar 7 para la preparación del ABP del próximo curso, dejando los restantes 5 procesos para ampliar en cursos siguientes. Los 7 procesos se seleccionaron en base a las posibilidades de planteamiento de problemas diferentes y por la experiencia y conocimiento de los profesores. En la Tabla 3 se muestran los procesos industriales finalmente elegidos. 
Tabla 3. Procesos industriales propuestos para el ABP

\begin{tabular}{ll}
\hline $\mathbf{N}^{\mathbf{0}}$ & Proceso industrial \\
\hline 1 & Obtención de sosa mediante el proceso SOLVAY \\
2 & Elaboración de zumos \\
3 & Obtención de biodiesel a partir de aceites usados \\
4 & Pintado de carrocerías de automóvil por cataforesis \\
5 & Obtención de aceite de orujo \\
6 & Digestión anaerobia de fangos \\
7 & Procesado del biogás para su aplicación posterior \\
\hline
\end{tabular}

Tras la selección de los procesos, cada profesor se ha encargado de plantear el enfoque de los posibles problemas, teniendo en cuenta los condicionantes ya descritos anteriormente, y que se resumen a continuación:

- Deben plantearse situaciones complejas que requieran análisis

- Se debe procurar cierto carácter multidisciplinar

- El problema debe requerir la búsqueda y selección de la información adecuada

- El resultado de aprendizaje debe contemplar la evaluación de la solución con criterios técnicos, económicos y/o sostenibles (medioambientales y de seguridad)

- Deben estar enfocados a las unidades didácticas 2, 4 y 5; para que puedan desarrollar las actividades de manera progresiva a lo largo del cuatrimestre.

Se acordó que cada profesor plantearía, al menos, 4 enfoques diferentes de problemas. Con ello se pretende contar con una batería significativa de problemas para cada proceso, de manera que cada profesor pueda aplicar el ABP centrado en 2-3 procesos máximo, lo que facilitará el seguimiento y resolución de dudas.

\subsection{Ejemplo de planteamiento del ABP en uno de los procesos propuestos}

En este apartado se describe uno de los problemas planteados, como ejemplo de resultado de la primera fase del Proyecto de Innovación.

El problema se engloba dentro del proceso industrial $n^{\circ} 4$, de los presentados en la Tabla 1: "Proceso de pintado de carrocerías de automóvil por cataforesis". En la Fig. 2 se muestra el diagrama de bloques general del proceso, similar al que los estudiantes deberán elaborar en el Entregable 1. En color rojo se ha resaltado la etapa del proceso objeto del problema que se muestra de ejemplo en la Tabla 4. 


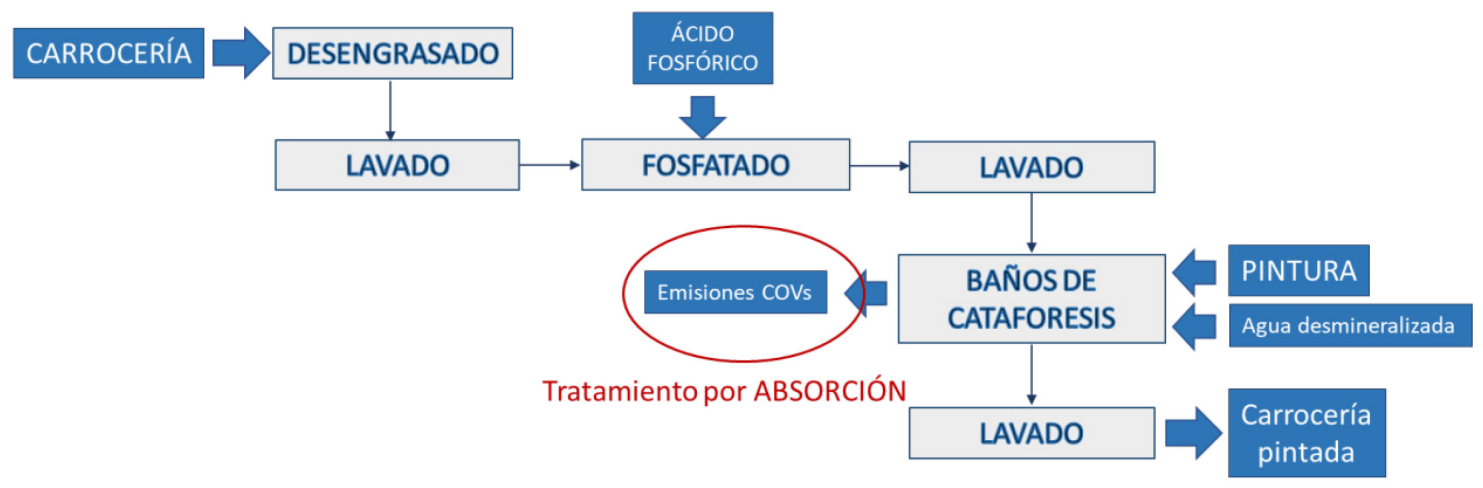

Fig. 2 Ejemplo de diagrama de bloques a elaborar por los estudiantes

El proceso planteado corresponde al pintado de carrocerías de automóvil mediante baños de cataforesis. En dicha etapa de pintado, debido al uso de disolventes en la pintura, se emiten Compuestos Orgánicos Volátiles (COVs), cuya emisión a la atmósfera está limitada por la legislación, ya que se trata de sustancias peligrosas para la salud. El enfoque del problema plantea el diseño de una torre de absorción, una de las operaciones de separación estudiadas en la Unidad Didáctica 5, para la reducción de la cantidad de COVs en la corriente de aire, antes de su emisión a la atmósfera. Por lo tanto, se trata de un problema con un enfoque sostenible desde el punto de vista medioambiental y de seguridad. Tal y como se observa en la Tabla 4, mediante la resolución de este problema se espera que los estudiantes alcancen los siguientes resultados de aprendizaje:

1) Selección del líquido absorbente más adecuado para llevar a cabo la operación, teniendo en cuenta criterios de seguridad, consultados a partir de las Fichas de Datos de Seguridad correspondientes. Este planteamiento integra además los conocimientos estudiados en la Unidad Didáctica 4.

2) Cálculo de la cantidad de líquido que se necesita en la operación para reducir la cantidad de COVs por debajo del límite establecido por la legislación. Para ello, los alumnos deberán buscar datos del equilibrio líquido-vapor de las fases definidas en el resultado anterior, así como consultar la legislación para conocer el límite de emisión de la actividad industrial objeto de estudio. Con todo ello, y los datos proporcionados, podrán hacer el cálculo pertinente.

3) Cálculo de la altura de torre necesaria para conseguir el propósito de la operación. En este caso, los estudiantes deberán consultar páginas web de casas comerciales suministradoras de rellenos industriales, para seleccionar el mismo y obtener el valor del factor de empaque suministrado por el fabricante, con el que podrán calcular la altura requerida. Finalmente, deberán contrastar si el valor de altura obtenido es coherente con las torres industriales. En caso contrario, se deberá seleccionar otro tipo de relleno y realizar de nuevo el cálculo.

Tabla 4. Ejemplo de problema propuesto para la aplicación del ABP

\begin{tabular}{|l|l|}
\hline Proceso & Pintado carrocerías por cataforesis \\
\hline Tipo de problema & Unidad Didáctica 5: ABSORCIÓN \\
\hline Enfoque & $\begin{array}{l}\text { Diseño de una torre de absorción para reducir la concentración de COVs } \\
\text { por debajo del límite de emisión }\end{array}$ \\
\hline Resultados esperados & $\begin{array}{l}\text { Selección del líquido absorbente más adecuado teniendo en cuenta } \\
\text { criterios de seguridad } \\
\text { Cálculo de la cantidad de líquido requerida (a partir de la condición de } \\
\text { pendiente mínima) } \\
\text { Cálculo de la altura de la torre }\end{array}$ \\
\hline
\end{tabular}




\begin{tabular}{|l|l|}
\hline \multirow{5}{*}{ Datos necesarios } & $\begin{array}{l}\text { Caudal de gas generado en el proceso de pintado } \\
\text { Diámetro de la torre } \\
\text { Concentración de COVs en el gas generado } \\
\text { Coeficiente de transferencia de materia de los COVs en el gas }\end{array}$ \\
\hline Información a buscar & $\begin{array}{l}\text { Absorbentes posibles } \\
\text { Equilibrio líquido-gas } \\
\text { Tipos de relleno } \\
\text { Factor de empaque } \\
\text { Límite de emisión de COVs para la actividad (RD 117/2003) }\end{array}$ \\
\hline
\end{tabular}

Una vez definida la metodología y la evaluación para implementar el ABP en la asignatura, y elaborados los problemas a resolver por los estudiantes, durante el curso 2020-21 se llevará a cabo la implementación de la innovación. El análisis de los resultados de la misma, está previsto realizarlo mediante las siguientes herramientas:

- Alcance de los resultados de aprendizaje, evaluados a través de los entregables y del seguimiento del trabajo progresivo de los estudiantes (actas de reuniones de trabajo y de reuniones de tutoría).

- Encuestas internas de satisfacción de los estudiantes con la innovación implementada.

- Test de enfoques de aprendizaje, que se contrastará con los resultados de dicho test pasado este curso 2019-20 al finalizar la asignatura.

\section{Conclusiones}

En este trabajo se han presentado los principales logros alcanzados en el primer curso de ejecución del Proyecto de Innovación que pretende mejorar el aprendizaje en los estudiantes de la asignatura "Tecnología Química Industrial", relativos a la definición de la metodología y la evaluación, y a la elaboración de los materiales didácticos. Dichos logros se resumen en los siguientes puntos:

- Se ha decidido que los alumnos se organizarán en equipos de trabajo de 4-5 miembros, de manera que cada grupo de teoría se dividirá en 8-10 equipos.

- La actividad ABP será obligatoria para todos los estudiantes y supondrá un $10 \%$ en la calificación final de la asignatura.

- La actividad consistirá en la resolución de dos problemas (uno en cada parcial) dentro de un mismo contexto, que corresponderá con un proceso industrial en el que se aplique la tecnología química.

- Los problemas estarán enfocados hacia la viabilidad económica de los procesos y/o su sostenibilidad, desde el punto medioambiental y de seguridad.

- Como resultado de la actividad, cada equipo de alumnos presentará 3 entregables a lo largo del cuatrimestre, además de 2 fichas con el planteamiento de cada problema para su revisión por el profesor, previa a la resolución de los mismos.

Por último, para completar las tareas planificadas para este curso se deberán incorporar las modificaciones pertinentes a la Guía Docente de la asignatura, así como elaborar todo el material necesario para la implementación de la innovación durante el siguiente curso 2020-21, cuyos resultados se analizarán tras la finalización de la asignatura, una vez se tengan las herramientas necesarias para ello: trabajo académico de los estudiantes, actas de reuniones y tutorías, encuesta interna de satisfacción y test de enfoques de aprendizaje. 


\section{Agradecimientos}

Este trabajo ha sido financiado por el VECA (Convocatoria A + D. Proyectos de Innovación y Mejora Educativa) de la Universitat Politècnica de València.

\section{Referencias}

BAYAT, S. y TARMIZIB, R.A. (2012). "Effects of problem-based learning approach on cognitive variables of university students" en Procedia - Social and Behavioral Sciences, vol. 46, p. 3146 - 3151.

BARROW, H.S. (1986). “A toxonomy of problem-based learning methods” en Medical Education, vol. 20 , p. 481 486.

BENLI, E. y MUSTAFA, S. (2012). "The investigation of the effect of problem based learning to the academic achievement and the permanence of knowledge of prospective science teacher: the problem of the boiler stone" en Procedia - Social and Behavioral Sciences, vol. 46, p. 4317 - 4322.

BIGGS, J. (1993). "What do inventories of students' learning processes really measure? A theoretical review and clarification", en British Journal of Educational Psychology, vol. 63, p. 3-19.

COSTANTINO, L. y BARLOCCO, D. (2019). "Teaching an Undergraduate Organic Chemistry Laboratory Course with a Tailored Problem-Based Learning Approach” en Journal of Chemical Education, vol. 96, issue 5, 888-894.

DE GRAAFF, E. y KOLMOS, A. (2003). “Characteristics of Problem-Based Learning” en Int. J. Engng. Ed., vol. 19, issue 5, p. 657-662.

DRĂGHICESCU, L.M.; PETRESCU, A.M.; CRISTEA, G.C.; GORGHIU, L.M. y GORGHIU, G. (2014). "Application of Problem-based Learning Strategy in Science Lessons - Examples of Good Practice" en Procedia Social and Behavioral Sciences, vol. 149, issue 5, p. 297-301.

DROBNIV VIDIC, A. (2008). "Development of Transferable Skills within an Engineering Science Context using Problem-Based Learning” en Int. J. Engng Ed., vol. 24, issue 6, p. 1071-1077.

FARIDA HARUNA, N.; MOHD YUSOF, K., ZAMRY JAMALUDIN, M. y SYED HASSAN, S.A.H. (2012). "Motivation in Problem-based Learning Implementation" en Procedia - Social and Behavioral Sciences, vol. 56, p. 233-242.

GARGALLO, B. (2008). "Estilos de docencia y evaluación de los profesores universitarios y su influencia sobre los modos de aprender de sus estudiantes” en Revista Española de Pedagogía, vol. 241, p. 425-445.

GARGALlO LÓPEZ, B.; GARCÍA FÉLIX, E.; MORERA BERTOMEU, I. y BENAVENT GARCÉS, A. (2015). "Métodos innovadores y enfoques de aprendizaje en estudiantes universitarios" en Congreso In-Red 2015, Valencia julio 2015.

LAU, H.Y.K. y MAK, K.L. (2004). "The virtual company: a re-configurable open shell for problem-based learning in industrial engineering” en Computers \& Industrial Engineering, vol.47, p. 289-312.

LENNON, D.; FREER, A.A.; WINFIELD, J.M.; LANDONA, P. y REIDB, N. (2002). “An undergraduate teaching initiative to demonstrate the complexity and range of issues typically encountered in modern industrial chemistry" en Green Chemistry, vol. 4, p. 181-187.

PÉREZ GRANADO, L. (2018). "El aprendizaje basado en problemas como estrategia didáctica en educación superior" en Voces de la educación, vol. 3, Nº 6, p. 155-167.

PERRENET, J.C.; BOUHUIJS, P.A.J. y SMITS, J.G.M.M. (2000). “The Suitability of Problem-based Learning for Engineering Education: Theory and practice" en Teaching in Higher Education, vol. 5, issue 3, p. 345-358.

VIZCANO GUARCH, C. y JUÁREZ, E. (2008). “QQué es y cómo funciona el aprendizaje basado en problemas?” en García Sevilla, J. El aprendizaje basado en problemas en la enseñanza universitaria. Sevilla: Universidad de Murcia, Servicio de Publicaciones. p. 17-36. 
María Sancho, Beatriz García-Fayos, Esperanza M. García-Castelló, Manuel César Marti-Calatayud, Antonio D. Rodríguez-López, Amparo Bes-Pia, José Antonio Mendoza-Roca y Asunción Santafé-Moros

WAI HUNG, I.P. y CHOI, A.C.K. (2003). “An Integrated Problem-Based Learning Model for Engineering Education” en Int. J. Engng. Ed., vol. 19, issue 5, p. 734-737.

WAI LAUA, Y. y YEE LIMB, S. (2015). "Learning approaches in accounting education: Towards deep learning” en Management Science Letters, vol. 5, p. 861-866. 